\title{
A Hospital-Based Prospective Cohort Study of Aged People to Elucidate the Predictive Factors for Chronic Disease and Mortality: HAPPY Cohort
}

\author{
Kyoung Min Kim ${ }^{1,2, *}$, Tae Jung $\mathrm{Oh}^{1,2, *}$, Hana $\mathrm{Jang}^{1}$, Hyun Jung Kook ${ }^{1}$, Min Seung Song ${ }^{1}$, Hee Youn Lee, \\ Hak Chul Jang ${ }^{1,2}$, Soo Lim ${ }^{1,2}$ \\ ${ }^{1}$ Department of Internal Medicine, Seoul National University Bundang Hospital, Seongnam, ${ }^{2}$ Department of Internal Medicine, Seoul \\ National University College of Medicine, Seoul, Korea
}

Corresponding Author:

Soo Lim, MD, PhD

Department of Internal

Medicine, Seoul National

University Bundang

Hospital, Seoul National

University College of

Medicine, 82 Gumi-ro

173beon-gil, Bundang-gu,

Seongnam 13620, Korea

\section{Tel: $+82-31-787-7035$}

Fax: +82-31-787-4051

E-mail: limsoo@snu.ac.kr

*These authors contributed equally to this study and should be considered co-first authors.

Received: June 5, 2017 Revised: June 6, 2017 Accepted: June 20, 2017
Background: The world over, the multimorbidity associated with aging is proving to be a great socio-economic burden. The loss of muscle mass and increased adiposity are typical of aging, but the number of longitudinal studies focusing on sarcopenia or sarcopenic obesity is currently limited. We launched a hospital-based, prospective cohort study of aged people (HAPPY cohort) to identify the impact of sarcopenia or sarcopenic obesity on the occurrence of chronic diseases including diabetes, or its vascular complications, osteoporosis and fractures, cognitive dysfunction, and mortality. Methods: For the HAPPY cohort, we aim to recruit 1,000 patients, aged 60 years and above, who visit a tertiary hospital from August 2015. Participants are scheduled to undergo repeated waves of assessment every 3 years; this will be done for 9 years or until death. Results: General information and medical histories are collected through personal interviews and validated using medical records. Participants' physical function is assessed by diverse functional assessments including the hand grip strength test, timed up and go test, and the chair rise test. The functional status, nutritional status, levels of depression, and cognitive function of the participants are also measured. The body compositions are assessed using dual energy X-ray absorptiometry and the bioelectrical impedance method. The biochemical parameters related with chronic diseases are measured. Conclusion: This HAPPY cohort study will provide results relevant to the many health issues among elderly Asians (among at least elderly Koreans). These results will help us gain a better understanding of sarcopenia and sarcopenic obesity, as well as the cross-sectional and longitudinal association of chronic diseases and mortality among elderly adults.

Key Words: Elderly, Aging, Cohort, Longitudinal study

\section{INTRODUCTION}

In recent times, Korea has been experiencing a rapid aging of its population, due to decreased birth rates and the extension of life expectancy ${ }^{1)}$. According to Statistics Korea, the population of adults aged 65 years or older was $9.1 \%$ in 2005 , and is expected to reach $15.7 \%$ in 2020 and $24.1 \%$ in 2030; this growth is the fastest among all the Organization for Economic Co-operation and Development countries ${ }^{2}$. The consequences of this increase in the elderly population include an increase in the social burden of chronic metabolic complications $^{2}$. In terms of medical expenses too, there has been a drastic increase in the medical expenses pertaining to the treatment of cerebrovascular and cardiovascular diseases ${ }^{3)}$.
Aging-related changes in body composition are reported to be negative, with the levels of body fat increasing constantly and the muscle mass and strength decreasing ${ }^{4)}$. These changes lead to various physical and physiological changes, and also affect physical function and the risk of various diseases ${ }^{5-10)}$. In terms of the reduction of muscle mass due to aging, the reduction to an extent that it leads to limited muscle strength or performance of physical activities is referred to as 'sarcopenia,' which has various negative effects on the health of the elderly. Therefore, it is imperative to accurately evaluate the current state of sarcopenia and explore various cures for it ${ }^{11,12)}$. In the United States, about $30 \%-40 \%$ of elderly adults, aged 70 years or older, have sarcopenia ${ }^{13,14)}$. Currently, countries around the world are working towards effectively identifying the actual status of 
sarcopenia, to establish a cohort of patients with myopathy, for the purpose of reducing the accompanying disability/ mortality ${ }^{15)}$. However, in Korea, the research being done on this topic is still insufficient.

Decreased muscle mass, due to aging, causes a decrease in muscle strength and thereby, an increase in the risk of falling, resulting in incidences of fractures of the femur ${ }^{16-18)}$. It has been reported that even after adjusting the various risk factors such as age, sarcopenia still increases the risk of falls by 2.5 times in elderly adults, especially elderly men ${ }^{7)}$. In addition, sarcopenia among the elderly is eventually the main cause of the increase in mortality ${ }^{19,20)}$.

Aging-related changes in the body composition are known to increase not only the risk of functional impairment and physical disability, but also the risk of metabolic disorders ${ }^{21)}$. The aging-related decline in muscle mass and increase in body fat mass/visceral fat are characteristic of sarcopenic obesity and are closely associated with increased risks of cardiovascular/cerebrovascular diseases, and type 2 diabetes mellitus ${ }^{22,23)}$.

Sarcopenic obesity among the elderly is consistent with the concept of metabolic diseases (diabetes mellitus/cardiovascular/cerebrovascular diseases), so it is called the 'Metabolically Obese but Normal Weight'. Sarcopenic obesity is more prevalent in the Asian population whose body weight is relatively normal compared to the westerners.

The increased growth rate of the elderly population in Korea and the association of sarcopenia/sarcopenic obesity with chronic diseases such as diabetes and cardiovascular/ cerebrovascular disease has led to an increase in the loss of socio-economic costs, due to the increased financial burden of appropriate treatment. This indicates that the need for studies focusing on the accurate diagnosis criteria, as well as the prevalence and etiology of sarcopenia/sarcopenic obesity is extremely high, so as to be able to develop appropriate therapeutic technologies for the treatment of elderly Koreans $^{24)}$.

Based on these epidemiologic trends and medical needs, we have launched a hospital-based, prospective longitudinal cohort of adults, aged 60 years or older, to comprehensively assess the impact of sarcopenia/sarcopenic obesity on chronic medical conditions and mortality. We plan to follow up on participants for 9 years, for long-term evaluation. This article aims to describe the study design and other details of the HAPPY aging cohort.

\section{MATERIALS AND METHODS}

\section{Aims of the Project}

The aim of our study is to investigate the cross-sectional and longitudinal association of sarcopenia/sarcopenic obesity with chronic diseases, including diabetes mellitus and its vascular complications, osteoporosis and fractures, cognitive dysfunction, and mortality among an elderly cohort. At the baseline, we will measure the clinical and biochemical risk factors relevant to sarcopenia/sarcopenic obesity.

\section{Study Design}

We have designed a hospital-based prospective cohort study. Participants are recruited at the Seoul National University Bundang Hospital (SNUBH), Seongnam City, South Korea. The study protocol has been approved by the Institutional Review Board of SNUBH (approval number: 1507306-306). All the participants will provide written informed consent before participation in the study, which has been registered at https://clinicaltrials.gov (ClinicalTrials.gov ID: NCT02640872)

\section{1) Inclusion criteria}

- Men and women over 60 years of age, capable of communication with the research team and functional assessment.

- Written and signed consent to participate in this cohort study.

\section{2) Exclusion criteria}

- Those who have received cancer treatment in the past 6 months (including, chemotherapy, radiation therapy, and surgery). Participants whose treatment for cancer ended 6 months prior to the start of the study can be included.

- Those with severe liver disease (Child Pugh class $C$ liver cirrhosis, decompensated liver disease), end-stage renal disease, and decompensated chronic renal failure (NYHA class IV).

- Those who are deemed unfit to undergo the evaluation of exercise performance as well as to respond to the questionnaire survey, due to severe cognitive impairment.

- Those who are newly diagnosed with thyroid dysfunction (within a span of 3 months prior to the study) and are starting medication therapy. (Cases in which there have been no changes in the dosage of thyroid hormone and antithyroid medications for a period of 3 months, and those do not have symptoms associated with thyroid dysfunction can be included).

- Those with acute diseases that require hospitalization.

- Those who are deemed unfit for participation in this study by the investigator.

\section{Target Sample Size and Justification}

This study is a prospective observational study that aims 
Table 1. Incidences of major diseases in Korea

\begin{tabular}{|c|c|c|c|}
\hline Name of disease & Year & Characteristics & Incidence rate \\
\hline Diabetes mellitus & 2004 & 60 Years or older & $2.9 \% / 3 \mathrm{yr}$ \\
\hline Hypertension & 2005 & Mean age, 65.8 years & $37.7 \%$ / $5 \mathrm{yr}$ \\
\hline Myocardial infarction & 2003 & Corrected by standard population distribution & $0.101 \%$ / $1 \mathrm{yr}$ \\
\hline Stroke & 2003 & Corrected by standard population distribution & $0.207 \%$ / $1 \mathrm{yr}$ \\
\hline Osteoporosis & 2012 & $\begin{array}{l}50 \text { Years or older } \\
\text { Corrected by standard population distribution }\end{array}$ & $\begin{array}{l}\text { Men: } 0.443 \% / 1 \mathrm{yr} \\
\text { Women: } 1.890 \% / 1 \mathrm{yr}\end{array}$ \\
\hline Vertebral fracture & 2012 & $\begin{array}{l}50 \text { Years or older } \\
\text { Corrected by standard population distribution }\end{array}$ & $\begin{array}{l}\text { Men: } 0.313 \% / 1 \mathrm{yr} \\
\text { Women: } 0.953 \% / 1 \mathrm{yr}\end{array}$ \\
\hline Hip fracture & 2011 & $\begin{array}{l}50 \text { Years or older } \\
\text { Corrected by standard population distribution }\end{array}$ & $\begin{array}{l}\text { Men: } 0.087 \% / 1 \mathrm{yr} \\
\text { Women: } 0.198 \% / 1 \mathrm{yr}\end{array}$ \\
\hline
\end{tabular}

to evaluate the relationship between the baseline index at the time of the participation in the study and the morbidity of chronic illnesses occurring during the follow-up period. In relation to the chronic diseases that are the outcome variables, including diabetes, hypertension, obesity, myocardial infarction and angina, cerebrovascular disease, osteoporosis, fracture, thyroid dysfunction, heart failure, chronic kidney disease, and chronic liver disease, a comprehensive study is to be performed.

Although there is plenty of data on the prevalence of chronic diseases in Korea, there is only a limited number of studies on their incidence rates. The incidences of each chronic disease in Korea are identified through the review of literature (Table 1). Based on this, the outcome event should have an incidence rate of $18.2 \%$ per 3 years to obtain statistical significance after the first follow-up observation for 3 years, and an incidence rate of $9.1 \%$ after the second follow-up observation for 6 years. Based on the aforementioned information, it is possible to verify the statistical significance of cases of hypertension with high incidence rates, but in the cases of myocardial infarctions or strokes with low incidence rates, it is difficult to achieve statistical significance. However, this study does not plan to limit itself to cardiovascular and cerebrovascular disease, and myocardial infarctions or strokes. One of the objectives of the study is also to analyze relevant diseases such as angina pectoris or transient ischemia. In addition, since we plan to combine various chronic diseases as listed in Table 1, and analyze them as the composite endpoint, it is considered that the limitation of statistical significance can be overcome.

The sample size of the present study was calculated as follows. Based on the incidence rate of major diseases in South Korea, we defined an $\alpha$-cutoff of $5 \%$ and a $\beta$-cutoff of $20 \%$. The exposure rate is estimated as 0.1 and relative hazard as 2.0. The total event is estimated to be 182. Consequently, the recommended number of study participants is $1,000(80 \%$ power, significance level $\mathrm{p}<0.05)$ (Clinical and Translational Science Institute, UCSF, http://www.sample-size.net) (Table 2).
Table 2. Sample size for survival analysis of the outcome event

\begin{tabular}{lc}
\hline \hline Variable & Value \\
\hline Two-tail power of test $(\alpha)$ & 0.05 \\
Power $(\beta)$ & 0.8 \\
Exposure rate of risk factors & 0.1 \\
Relative hazard & 2.0 \\
Total event needed & 182
\end{tabular}

Adapted from Clinical and Translational Science Institute, UCSF, http://www.sample-size.net.

\section{Study Participant Recruitment Plan}

The participants who meet the inclusion criteria are recruited after the overview and purpose of the study are explained to them. The principal investigator and the sponsor do not rule out the participation of patients based solely on their race or socio-economic status. We try to enable patients to participate in this study, as long as they meet the study's inclusion criteria.

The first phase of the study will take place over 3 years, which will serve as the first quarter, and recruitment for the participants for the first year of the first quarter will begin. If a patient agrees to participate in the study and is selected, a questionnaire survey will be conducted to evaluate the Physical Activity Scale, which includes medical history, biomarkers, muscle strength, exercise ability, body composition and cognitive function. Thereafter, each participant will visit the hospital in 3-year intervals and be tested for the predictors of morbidity or the exacerbation of chronic illness, through changes in the various indicators and the measurement of indices.

\section{Observation Items, Clinical Test Tems, and Observational Examination Method}

Measures assessed at the baseline will be repeated in every evaluation, as shown in Table 3. 
Table 3. Measurement factors in the HAPPY cohort

\begin{tabular}{|c|c|}
\hline Research items & Assessment \\
\hline Muscle strength assessment & Hand grip strength \\
\hline Motor ability assessment & 4-Min walking test, Chair stand test, Timed Up and Go test \\
\hline \multicolumn{2}{|l|}{ Questionnaire survey } \\
\hline Cognitive function assessment & MMSE-K, IQCODE \\
\hline Activities of daily living or physical activities & K-ADL, K-IADL, K-PASE \\
\hline Quality of life & SF-36 \\
\hline Depression screening & PHQ-9 \\
\hline \multicolumn{2}{|l|}{ Biochemistry } \\
\hline Blood test & $\begin{array}{l}\text { CBC, Fasting glucose, BUN, Creatinine, Total protein, Albumin, alkaline phosphatase, } \\
\text { AST, ALT, Total bilirubin, Calcium, Phosphorus, Electrolyte, Triglyceride, HDL, } \\
\text { LDL, HbA1c, Insulin, TSH, Free T4, hsCRP, 25(OH)-vitamin D, Parathyroid hor- } \\
\text { mone, Osteocalcin, C-telopeptide }\end{array}$ \\
\hline Blood sample stock & EDTA $(3 \mathrm{~mL})$, SST $(6 \mathrm{~mL})$ \\
\hline Urinalysis & Urine microalbumin/creatinine ratio \\
\hline Body composition & Bioelectrical impedance analysis, Whole body dual energy X-ray absorptiometry \\
\hline Bone mineral density & Dual energy X-ray absorptiometry \\
\hline \multicolumn{2}{|c|}{$\begin{array}{l}\text { MMSE-K, Mini Mental Status Examination; IQCODE, informant questionnaire on cognitive decline in the elderly; K-ADL, Korean } \\
\text { activities of daily living; K-IADL, Korean instrumental activities of daily living; K-PASE, Korean Physical Activity Scale for the Elderly; } \\
\text { SF-36, 36-Item Short Form Health Survey; PHQ-9, Patient Health Questionnaire-9; CBC, complete blood cell; BUN, blood urea } \\
\text { nitrogen; AST, aspartate aminotransferase; ALT, alanine aminotransferase; HDL, high-density lipoprotein; LDL, low-density lipopro- } \\
\text { tein; HbA1c, hemoglobin A1C; TSH, thyroid-stimulating hormone; hsCRP, high-sensitivity C-reactive protein; EDTA, ethylenediamine- } \\
\text { tetraacetic acid; SST, serum separate tube. }\end{array}$} \\
\hline
\end{tabular}

\section{1) Baseline Study}

\section{(1) Demographics and anthropometric measurements}

At the baseline examination, the demographics, general health status, and social history such as alcohol consumption, smoking status, physical activity, and any family history of chronic diseases are assessed using self-completed questionnaires. We define ex-smokers as those who have smoked more than 100 cigarettes in their lifetime, but who do not smoke at present. Physical activity is divided into 4 categories: (1) none, (2) regular walking, which is defined as walking -30 min per day, at least 3 days a week, (3) regular exercise, which includes activities such as fast walking, tennis, regular cycling, and moderate-intensity daily activities such as mopping the floor $\geq 30 \mathrm{~min}$ a day, at least 3 days a week, (4) vigorous exercise, which includes running, fast cycling, and climbing $>20$ min a day, at least 3 days a week. Participants are asked to wear light indoor clothing when their height and weight are measured using a distal electronic scale. The body mass index is calculated by dividing the weight $(\mathrm{kg})$ by the height squared $\left(\mathrm{m}^{2}\right)$. The waist circumference is measured at the midline between the lowest rib margin and the iliac crest, using a measuring tape. The hip circumference is measured at the maximum protuberance of the buttocks, and the waist-hip ratio (WHR) is calculated. The thigh circumference is measured at the mid-thigh level, be- tween the inguinal crease and the proximal border of the patella. Systolic and diastolic blood pressures are measured with an electronic blood pressure meter (HEM-7111, Omron Healthcare Co., Ltd., Kyoto, Japan) when the participants are seated. Values for blood pressures are measured after 5 -minute periods of rest at a heart-level with standardized cuff in a sitting position, and measured twice 5 minute apart and the mean value is used in the analysis.

\section{(2) Physical function and performance}

To assess physical function, a 4-m walking test, chair rise test and Timed Up and Go test are conducted. The tests are first conducted on the investigators, after which they are conducted on the participants, who are instructed to do their best. Participants are allowed to have rest intervals of at least 5 minutes between the tests. The success/failure and total time to complete the tests (in seconds) are recorded using a stopwatch.

\section{(3) Hand grip strength measurement for muscle strength assessment}

Grip strength is evaluated using a dynamometer (Takei Scientific Instruments Co. Ltd., Tokyo, Japan) in kg. Participants' dominant arms are kept in a resting position, with the elbow angled properly on a desk, and then, they are asked to grip the dynamometer as tightly as they can, for a maximum of 3 seconds, after which they are allowed to 
relax their grip. The same test is then conducted for the nondominant arm. This test is repeated again after a 1to 2-min interval, in the order of the dominant arm and non-dominant arm.

\section{(4) Functional status and physical activities}

The functional status is assessed using 2 types of questionnaires: the Korean activities of daily living (K-ADL) and the Korean instrumental activities of daily living (K-IADL) which have been validated in previous studies ${ }^{25,26)}$. The K-ADL comprises basic everyday activities including dressing, bathing, eating, walking, and using the toilet, with 3 levels of independence (manage without help, need some help, and need considerable amount of help). The K-IADL consists of 10 questionnaires pertaining to shopping, using a phone, using public transportation, doing light house work, doing laundry, mobility outdoors, preparing meals, taking medicine, and managing money. The levels of the responses are divided into 3 or 4 levels in each question, according to the degree of dependency. In addition, the Physical Activity Scale is assessed using the Korean Physical Activity Scale for the Elderly (K-PASE) ${ }^{27)}$.

\section{(5) Cognitive function}

To evaluate the cognitive function, 2 types of questionnaires are given to all the participants: the Korean version of the Informant Questionnaire on Cognitive Decline in the Elderly (IOCODE) and the Mini-Mental State Examination for dementia screening (MMSE-KC) ${ }^{28,29)}$. The IOCODE consists of 26 questions which assess memory and intelligence.

\section{(6) Depression}

Depressive symptoms are assessed using the Patient Health Questionnaire-9 $(\mathrm{PHO}-9)^{30}$. We administered the Korean version of the PHQ-9 $9^{31}$. The PHQ-9 is a shorter version of the $\mathrm{PHO}$, and consists of a 9-item depression module. Each of the 9 items can be scored from 0 to 3 , according to the number of days, in the past 2 weeks, in which participants have had depressive symptoms, loss of interest, sleep disorders and suicidal desires. The severity of the depressive symptoms is calculated as a sum of the scores in each item, ranging from 9 to 27 .

\section{(7) Nutritional analysis}

The 3-day food record method is used to assess participants' nutritional status. All the participants in this study are asked to complete a 3-day food record (2 weekdays and 1 day, on a weekend) in the baseline evaluation and the follow-up visit. A trained nutritionist later evaluates the intakes and the intake amounts through an interview. The 3-day food records are analyzed using the CAN-Pro 5.0, a computer-aided nutritional analysis program developed by the Korean Nutrition Society (Seoul, Korea).

\section{(8) Bioelectrical impedance analysis (BIA) for body composition}

After overnight fasting, the body composition of all the participants (they are asked to wear light clothing) is measured after urination, by a trained nurse, using a BIA machine (Inbody770, InBody, Seoul, Korea), on the same day as the blood sampling. The water intake is restricted before each BIA measurement. At first, participants are placed in an upright position with bare feet, and the instructors enter their identification number, age, sex, and height into the machine. The participants are then instructed to grasp the electrode bars with both hands and to keep both arms at a distance from the trunk. Eight electrodes contact the participants' feet and hands. After the machines are turned on by the instructors, 6 different frequencies $-1,5,50,250,500$, and $1,000 \mathrm{kHz}$ - are used on 5 segments of the body: both arms and legs, and the trunk.

(9) Dual energy X-ray absorptiometry for body composition and bone mineral density

Whole-body composition measurement is performed using dual energy X-ray absorptiometry (DXA). All the scans have been acquired in accordance with the manufacturers' standard scan and positioning protocols. Images are analyzed using the whole-body software module (ODR v.12.3, Hologic Inc., Bedford, MA, USA). Values pertaining to the total fat and lean mass of the whole body or at each region are obtained. The visceral fat area is also calculated. The bone mineral density is measured at the lumbar spine (L1-L4), femoral neck, and total hip, using DXA (DXA, Discovery W; Hologic Inc., Bedford, MA, USA). The left hip is scanned routinely, but in participants with a left hip fracture or device, the right hip is scanned. The DXA data are analyzed using Hologic APEX software version 5.5.3 in its default configuration.

\section{(10) Biochemical data}

We instruct participants not to eat food after midnight, on the night before testing. Baseline blood samples are drawn after at least 8 hours of fasting and centrifuged at 3,000 rpm for 10 minutes, at $4^{\circ} \mathrm{C}$. The plasma levels of glucose are analyzed using the hexokinase method. High-density lipoprotein cholesterol and low-density lipoprotein cholesterol, and triglyceride levels are measured by homogeneous enzymatic assays and glycerol-3-phosphate oxidase peroxide methods, respectively. An XE-2100 Hematology Analyzer is used for measuring the white blood cell count, hemoglobin level, hematocrit, and platelet count. Aspartate and alanine aminotransferase (NADH-UV method), total bilirubin (bilirubin oxidase method), thyroid function test (electrochemiluminescence immunoassay), blood urea nitrogen (urease/GLDH 
method), creatinine (Jaffe's kinetic method), calcium (OC/PC method), and phosphorus (Molybdate reduction method) are measured at the central laboratory of SNUBH, as reported previously ${ }^{32,33)}$. The biochemical tests are done immediately after sample collection. Aliquots of serum, plasma, whole blood, DNA, and RNA (PAX gene blood RNA system, Qiagen, Hilden, Germany) are kept in deep freezers until tested $\left(-80^{\circ} \mathrm{C}\right)$. All the parameters that we will investigate are shown in Table 3.

\section{2) Follow-up visit: in 3-year intervals}

Physical examinations, body composition tests, bone density tests, muscle strength assessments, motor ability assessments, questionnaire surveys, blood sampling tests, and urine tests will be performed as performed at the baseline visit.

The medical history of cases of morbidity associated with new chronic diseases will be investigated in the period between the participation in the cohort and the follow-up observation 3 years later. The chronic diseases include diabetes mellitus, hypertension, obesity, myocardial infarction and angina, cerebrovascular disease, osteoporosis, fracture, thyroid dysfunction, heart failure, chronic kidney disease, and chronic liver disease. The number of visits to the hospital, date of visit, and whether or not to take any medication will be also investigated.

\section{Data Analysis and Statistical Analysis Method}

All data are expressed as the mean and standard deviation, or as numbers and percentages. The Kolmogorov-Smirnov test is applied to evaluate the normality of variable distributions. In the case of continuous variables that are not normally distributed, nonparametric analysis is used. Before comparing the variables of the 2 genders, the differences in variances are subjected to Levene's test. The correlations between the variables are analyzed using the Pearson correlation coefficient. Categorical variables are compared using chi-square tests. Comparisons between means are analyzed using Student t-tests or nonparametric t-tests for continuous variables.

Survival analysis is performed based on the Cox-proportional hazards model, in which the factors examined at the baseline visit are considered potential risk factors and the assessment endpoints occurring between the baseline visit and the follow-up observation are used as dependent variables. Further, whether the potential risk factors act as independent risk factors will be examined on single or multivariate analysis. In the first univariate analysis, the hazard ratio is calculated for each risk factor. In the second multivariate analysis, it is conducted including the significant risk factors and the risk factors that have bases to clinically consider in the first univariate analysis. The analyses are performed using IBM SPSS Statistics for Windows, version 20.0 (IBM Corp., Armonk, NY, USA). For all the tests, $p<0.05$ is consid- ered to be statistically significant.

\section{RESULTS}

In this study's hospital-based cohort of the elderly, 1,000 Korean participants aged $\geq 60$ years will be recruited. The body composition, including the whole-body muscle and fat mass, will be assessed. The bone mineral density will be also examined. Biochemistry related with glucose and lipid metabolism will be measured.

\section{DISCUSSION}

South Korea is faced with the rapid aging of its population". And, there is a huge necessity for cohort studies, done on the elderly population, to identify the actual situation. In Korea, in particular, it is important to identify the actual conditions of sarcopenia and sarcopenic obesity among elderly adults. The definition and criteria of sarcopenia among elderly adults in Korea have not yet been established; in particular, every study presents the prevalence differently, as each of them used different diagnostic criteria and measurement methods, with discrepancies in the diagnostic indices $^{24,34,35)}$. Major countries, the world over, have already set standard values, taking into consideration racial ethnicities and continue to announce the prevalence rate using those values ${ }^{36)}$. However, considering the fact that there are differences in muscle mass between different racial ethnicities, as shown in the existing study, the limits of the values should be set differently according to the racial ethnicities. Thus, it is important to set a standard value corresponding to the actual conditions of Korea, and to identify the accurate status of sarcopenia based on the standard value in Korea.

We also plan to investigate the correlation between obesity and chronic metabolic diseases such as diabetes mellitus and cardiovascular/cerebrovascular diseases that are induced by sarcopenia/sarcopenic obesity in elderly Korean adults. The representative changes in body composition, brought on by aging and characterized by sarcopenia and the increase in body fat mass that are present together, are predicted to be major causes behind the increase in the incidences of cardiovascular/cerebrovascular diseases and senile metabolic diseases, as well as major causes of death and cancer ${ }^{23,37)}$. Therefore, it is necessary to confirm the role of sarcopenia or sarcopenic obesity in causing diabetes and cardiovascular/cerebrovascular diseases, among elderly Koreans. Most of the studies investigated so far are cross-sectional in design, and this is not sufficient to accurately determine the precursors of causative diseases. Therefore, it is required to elaborate on the precise risk level for each disease associated with sarcopenia/sarcopenic obe- 
sity, and analyze the causatively associated factors by establishing a long-term cohort study in the elderly.

Finally, we will elucidate on the causative factors and the development of prevention methods. In a rapidly aging society, the increase in the medical expenses of the elderly is a major burden on the national economy ${ }^{2}$. In order to reduce this burden, it is necessary to establish a healthy aging model. And, in order to construct a healthy aging model, it is imperative to identify the risk factors and inducers of sarcopenia and sarcopenic obesity. For such identification, it is necessary to analyze various factors such as the individuals' exercise frequency and intensity, body composition and biomarkers, and to confirm their association with disease occurrence or mortality through a long-term, prospective cohort study.

This study puts forth several points that differ from those of previous studies. The Korea Longitudinal Study of Health and Aging (KLOSHA) study in which our hospital has been the leading participant is representative of the Korean cohort study in the aged people ${ }^{38)}$. Although the KLOSHA was conducted in 1,000 elderly people in the community, it has the disadvantage of lower follow-up rate, and the morbidity rates of chronic diseases presented by it is also lower than those of the hospital cohort.

In addition, the number of large-scale studies pertaining to the elderly is extremely limited in Korea, and studies, the world over, on the predictive factors of chronic disease in elderly adults, are rare. This study performs the DXA test, which is considered as the gold standard in the measurement of bone mineral density and body composition, including fat mass and muscle mass. We also assess cognitive function, mental health, and the quality of life using standard questionnaires, so there is the advantage of being able to study the relationship between these factors and the health of the elderly adults.

This study has no criteria for discontinuation unless a patient withdraws his/her consent. In the follow-up observation study, the patient will be contacted primarily by telephone or else, by mail, if he/she does not show up at the hospital during the scheduled follow-up period. If participants cannot be reached, or if they refuse to visit the hospital, they will be dropped from the study. As for the cases of patient death, we will collect the data and collectively confirm the death through the survey report of the Statistics Korea on the Death Statistics.

In conclusion, the HAPPY cohort is a well-organized prospective cohort study focusing on body composition and its relationship with chronic health conditions in the elderly Korean population. Although our cohort is not communitybased, hospital-based cohorts have the advantage of providing detailed information about comorbidities, convenience in follow-up, and ease of continuing data collection. Regular follow-ups with the same protocols, in 3-year intervals, will help us expand on the current findings and enable us to dissect the possible factors of pathologic aging, in this cohort.

Conflicts of Interest Disclosures: The researchers claim no conflicts of interest.

\section{Acknowledgments}

This study was supported by a grant from the SNUBH (04-2013-002 and 10-2015-019), Dalim Biotech (06-2016-179), and Ildong (06-2016-079). This study was also supported by research funding from the Korean Geriatric Society.

\section{REFERENCES}

1. Kontis V, Bennett JE, Mathers CD, Li G, Foreman K, Ezzati M. Future life expectancy in 35 industrialised countries: projections with a Bayesian model ensemble. Lancet 2017;389:132335.

2. Organization WH. World report on ageing and health. Geneva: World Health Organization; 2015.

3. Kim J, Son M. National health insurance statistical yearbook. Seoul: Health Insurance Review and Assessment Service and $\mathrm{Na}$ tional Health Insurance Service; 2014.

4. Cheng Q, Zhu X, Zhang X, Li H, Du Y, Hong W, et al. A cross-sectional study of loss of muscle mass corresponding to sarcopenia in healthy Chinese men and women: reference values, prevalence, and association with bone mass. J Bone Miner Metab 2014;32:78-88.

5. Tyrovolas S, Koyanagi A, Olaya B, Ayuso-Mateos JL, Miret M, Chatterji $S$, et al. The role of muscle mass and body fat on disability among older adults: a cross-national analysis. Exp Gerontol 2015;69:27-35.

6. Miljkovic I, Kuipers AL, Cauley JA, Prasad T, Lee CG, Ensrud $\mathrm{KE}$, et al. Greater skeletal muscle fat infiltration is associated with higher all-cause and cardiovascular mortality in older men. J Gerontol A Biol Sci Med Sci 2015;70:1133-40.

7. McGrath R, Vincent BM, Al Snih S, Markides KS, Peterson $\mathrm{MD}$. The association between muscle weakness and incident diabetes in older Mexican Americans. J Am Med Dir Assoc 2017; 18:452.e7-452.e12.

8. Karakelides H, Nair KS. Sarcopenia of aging and its metabolic impact. Curr Top Dev Biol 2005;68:123-48.

9. Landi F, Liperoti R, Russo A, Giovannini S, Tosato M, Capoluongo E, et al. Sarcopenia as a risk factor for falls in elderly individuals: results from the ilSIRENTE study. Clin Nutr 2012; 31:652-8.

10. Roubenoff R. Sarcopenia and its implications for the elderly. Eur J Clin Nutr 2000;54 Suppl 3:S40-7.

11. Bouchard DR, Dionne IJ, Brochu M. Sarcopenic/obesity and physical capacity in older men and women: data from the Nutrition as a Determinant of Successful Aging (NuAge)-the Quebec longitudinal Study. Obesity (Silver Spring) 2009;17:2082-8.

12. Narici MV, Maffulli N. Sarcopenia: characteristics, mechanisms and functional significance. Br Med Bull 2010;95:139-59. 
13. Baumgartner RN, Koehler KM, Gallagher D, Romero L, Heymsfield SB, Ross RR, et al. Epidemiology of sarcopenia among the elderly in New Mexico. Am J Epidemiol 1998;147:755-63.

14. Janssen I, Heymsfield SB, Ross R. Low relative skeletal muscle mass (sarcopenia) in older persons is associated with functional impairment and physical disability. J Am Geriatr Soc 2002;50: 889-96.

15. Shafiee G, Keshtkar A, Soltani A, Ahadi Z, Larijani B, Heshmat R. Prevalence of sarcopenia in the world: a systematic review and meta- analysis of general population studies. J Diabetes Metab Disord 2017;16:21.

16. Tarantino U, Baldi J, Celi M, Rao C, Liuni FM, Iundusi R, et al. Osteoporosis and sarcopenia: the connections. Aging Clin Exp Res 2013;25 Suppl 1:S93-5.

17. Edwards MH, Dennison EM, Aihie Sayer A, Fielding R, Cooper C. Osteoporosis and sarcopenia in older age. Bone 2015;80: 126-30.

18. Cederholm T, Cruz-Jentoft AJ, Maggi S. Sarcopenia and fragility fractures. Eur J Phys Rehabil Med 2013;49:111-7.

19. Moon JH, Kim KM, Kim JH, Moon JH, Choi SH, Lim S, et al. Predictive values of the new sarcopenia index by the Foundation for the National Institutes of Health Sarcopenia Project for mortality among older Korean adults. PLoS One 2016;11:e 0166344.

20. Batsis JA, Mackenzie TA, Emeny RT, Lopez-Jimenez F, Bartels S. Low lean mass with and without obesity, and mortality: results from the 1999-2004 national health and nutrition examination survey. J Gerontol A Biol Sci Med Sci 2017 Feb 16 [Epub]. https://doi.org/10.1093/gerona/glx002.

21. Kim TN, Park MS, Lim KI, Choi HY, Yang SJ, Yoo HJ, et al. Relationships between sarcopenic obesity and insulin resistance, inflammation, and vitamin D status: the Korean Sarcopenic Obesity Study. Clin Endocrinol (Oxf) 2013;78:525-32.

22. Srikanthan P, Karlamangla AS. Relative muscle mass is inversely associated with insulin resistance and prediabetes. Findings from the third National Health and Nutrition Examination Survey. J Clin Endocrinol Metab 2011;96:2898-903.

23. Atkins JL, Whincup PH, Morris RW, Lennon LT, Papacosta O, Wannamethee SG. Sarcopenic obesity and risk of cardiovascular disease and mortality: a population-based cohort study of older men. J Am Geriatr Soc 2014;62:253-60.

24. Kim KM, Lim S, Choi KM, Kim JH, Yu SH, Kim TN, et al. Sarcopenia in Korea: prevalence and clinical aspects. J Korean Geriatr Soc 2015;19:1-8.

25. Won CW, Yang KY, Rho YG, Kim SY, Lee EJ, Yoon JL, et al. The Development of Korean Activities of Daily Living (K-ADL) and Korean Instrumental Activities of Daily Living (K-IADL) Scale. J Korean Geriatr Soc 2002;6:107-20.

26. Won CW, Rho YG, SunWoo D, Lee YS. The Validity and Relia- bility of Korean Instrumental Activities of Daily Living (K-IADL) Scale. J Korean Geriatr Soc 2002;6:273-80.

27. Choe MA, Kim J, Jeon MY, Chae YR. Evaluation of the Korean Version of Physical Activity Scale for the Elderly (K-PASE). Korean J Women Health Nurs 2010;16:47-59.

28. Kim TH, Jhoo JH, Park JH, Kim JL, Ryu SH, Moon SW, et al. Korean version of mini mental status examination for dementia screening and its' short form. Psychiatry Investig 2010;7: $102-8$.

29. Lee DW, Lee JY, Ryu SG, Cho SJ, Hong CH, Lee JH, et al. Validity of the Korean Version of Informant Questionnaire on the Cognitive Decline in the Elderly (IQCODE). J Korean Geriatr Soc 2005;9:196-202.

30. Kroenke K, Spitzer RL, Williams JB. The PHQ-9: validity of a brief depression severity measure. J Gen Intern Med 2001;16: 606-13.

31. Choi HS, Choi JH, Park KH, Joo KJ, Ga H, Ko HJ, et al. Standardization of the Korean Version of Patient Health Questionnaire-9 as a screening instrument for major depressive disorder. J Korean Acad Fam Med 2007;28:114-9.

32. Lim S, Kim KM, Kim MJ, Woo SJ, Choi SH, Park KS, et al. The association of maximum body weight on the development of type 2 diabetes and microvascular complications: MAXWEL study. PLoS One 2013;8:e80525.

33. Ku EJ, Jung KY, Kim YJ, Kim KM, Moon JH, Choi SH, et al. Four-year durability of initial combination therapy with sitagliptin and metformin in patients with type 2 diabetes in clinical practice; COSMIC study. PLoS One 2015;10:e0129477.

34. Kim KM, Lim S, Choi SH, Kim JH, Shin CS, Park KS, et al. Cardiometabolic implication of sarcopenia: The Korea National Health and Nutrition Examination Study (KNHANES) 20082010. IJC Metab Endocr 2014;4:63-9.

35. Kim KM, Jang HC, Lim S. Differences among skeletal muscle mass indices derived from height-, weight-, and body mass indexadjusted models in assessing sarcopenia. Korean J Intern Med 2016;31:643-50.

36. Studenski SA, Peters KW, Alley DE, Cawthon PM, McLean RR, Harris TB, et al. The FNIH sarcopenia project: rationale, study description, conference recommendations, and final estimates. J Gerontol A Biol Sci Med Sci 2014;69:547-58.

37. Lim S, Kim JH, Yoon JW, Kang SM, Choi SH, Park YJ, et al. Sarcopenic obesity: prevalence and association with metabolic syndrome in the Korean Longitudinal Study on Health and Aging (KLoSHA). Diabetes Care 2010;33:1652-4.

38. Moon JH, Lim S, Han JW, Kim KM, Choi SH, Kim KW, et al. Serum 25-hydroxyvitamin D level and the risk of mild cognitive impairment and dementia: the Korean Longitudinal Study on Health and Aging (KLoSHA). Clin Endocrinol (Oxf) 2015; $83: 36-42$. 